\title{
Field evaluation of the 22 rapid diagnostic tests for community management of malaria with artemisinin combination therapy in Cameroon
}

Innocent M. Ali ${ }^{1}, 2$, Jude D. Bigoga ${ }^{1}$, Dorothy A. Forsah ${ }^{3}$, Fidelis Cho-Ngwa ${ }^{4}$, Vivian Tchinda ${ }^{5}$, Vicky Ama Moor ${ }^{6}$, Josephine Fogako', Philomena Nyongalema', Theresa Nkoa ${ }^{7}$ Albert Same-Ekobo ${ }^{8}$, Joseph Mbede ${ }^{9}$, Etienne Fondjo ${ }^{2}$, Wilfred F. Mbacham ${ }^{1}$ and Rose G. F. Leke . $^{10^{*}}$

\begin{abstract}
Background: All suspected cases of malaria should receive a diagnostic test prior to treatment with artemisininbased combinations based on the new WHO malaria treatment guidelines. This study compared the accuracy and some operational characteristics of 22 different immunochromatographic antigen capture point-of- malaria tests (RDTs) in Cameroon to inform test procurement prior to deployment of artemisinin-based combinations for malaria treatment.

Methods: One hundred human blood samples (50 positive and 50 negative) collected from consenting febrile patients in two health centres at Yaoundé were used for evaluation of the 22 RDTs categorized as "Pf Only" (9) or "Pf + PAN" (13) based on parasite antigen captured [histidine rich protein II (HRP2) or lactate dehydrogenase (pLDH) or aldolase]. RDTs were coded to blind technicians performing the tests. The sensitivity, specificity, and predictive values of the positive and negative tests (PPV and NPV) as well as the likelihood ratios were assessed. The reliability and some operational characteristics were determined as the mean values from two assessors, and the Cohen's kappa statistic was then used to compare agreement. Light microscopy was the referent.

Results: Of all RDTs tested, $94.2 \%$ (21/22) had sensitivity values greater than $90 \%$ among which 14 (63.6 \%) were 'Pf + PAN'RDTs. The specificity was generally lower than the sensitivity for all RDTs and poorer for "Pf Only" RDTs. The predictive values and likelihood ratios were better for non-HRP2 analytes for "Pf + PAN" RDTs. The Kappa value for most of the tests obtained was around $67 \%$ (95 \% Cl 50-69\%), corresponding to a moderate agreement.

Conclusion: Overall, $94.2 \%$ (21/22) of RDTs tested had accuracy within the range recommended by the WHO, while one performed poorly, below acceptable levels. Seven "Pf + PAN" and 3 "Pf Only" RDTs were selected for further assessment based on performance characteristics. Harmonizing RDT test presentation and procedures would prevent mistakes of test performance and interpretation.
\end{abstract}

Keywords: Malaria, Sensitivity, Rapid diagnostic test, Microscopy, Specificity, HRP2, Positive predictive value, Plasmodium falciparum

\footnotetext{
*Correspondence: roseleke@yahoo.com

${ }^{10}$ Laboratory of Immunology and Parasitology, The Biotechnology

Centre, University of Yaoundé 1, BP 8094, Yaoundé, Cameroon

Full list of author information is available at the end of the article
} 


\section{Background}

The challenge of controlling malaria is a continuous reality in most sub-Saharan countries. Despite increasing efforts in prevention and treatment, malaria has remained a major cause of morbidity and mortality, with an estimated 451 million clinical cases of malaria in 2007 alone, mostly in sub-Saharan Africa [1]. In Cameroon, malaria continues to be endemic with estimated $71 \%$ living in high transmission areas [2], and the major cause of morbidity and mortality among the most vulnerable groups, namely children under 5 years of age and pregnant women, as well as the poor. The continuous existence of such huge burden due to malaria in 2010 has necessitated the implementation of large scale programs geared at eliminating malaria as a public health problem. Effective treatment of malaria requires precise laboratory diagnosis and this remains a cornerstone for global malaria control efforts. Microscopy still remains the method of choice in the diagnosis of malaria in endemic areas because it is cost effective. However correct identification of Plasmodium species by microscopy depends on factors, such as the experience of the microscopists, proper staining of the slides, good quality reagents, appropriate maintenance of the microscope and the time spent reading a slide. Due to these limitations, physicians are often reluctant to accept results of microscopy in such operational settings. Therefore, the reliance of diagnosis on clinical grounds alone has resulted in over diagnosis of malaria in many clinical settings in developing countries including Cameroon [3-5]. Rapid diagnostic tests have considerable potential as a tool to improve the diagnosis of malaria in endemic settings $[5,6]$. Due to the rapidity of the test and availability of the results for clinical care, RDTs have been positively recommended by the World Health Organization (WHO) when reliable microscopy is not available [7].

There is increasing interest in introducing RDTs for diagnosing malaria and improving malaria case management with artemisinin-based combination therapy (ACT), as studies show significant improvements in malaria control following implementation [8]. In 2010, forty-two African countries reported deployment of 11 million ACT at community level, with only a small proportion in the community receiving parasite confirmation test at community level [2]. Clearly, the WHO underscores the importance of expanding malaria diagnosis at community level as demonstrated in Zambia with scaling up of RDT use during the period 20042009 [2]. The benefits of such a strategy include reduction in expenditures on anti-malarial drugs, improved patient outcomes for non-malarial fevers and curbing of drug resistance $[15,16]$. The Government of Cameroon planned to introduce RDTs into communities in 50 pilot
Health Districts in the national territory in 2011 as part of a Global Fund supported project for deploying ACT for case management at community level. However, there has been little or no previous report on the field performance of different RDTs recommended by the WHO and available in the Cameroon market to properly inform procurement. It was, therefore, necessary to evaluate the performance of RDTs available in the Cameroon market. This study therefore aimed to determine and compare the diagnostic accuracy of 22 different rapid diagnostic tests available in Cameroon at that time.

\section{Methods \\ Participants}

The study population was made up of male and female febrile patients aged 1 year to 16 years suspected of a malaria infection in six busy peripheral health centres in Yaoundé. A patient was considered for enrolment into the study if he/she fulfilled the following criteria: willingness to donate $5 \mathrm{mls}$ of blood after informed consent. If the patient sample was positive by microscopy, consent/ assent was requested to participate. If the patient's blood sample was negative by microscopy, the patient was still requested to consent. This procedure was followed consecutively until the required number of malaria positive and negative samples was obtained. Those performing the microscopy at the health centres were trained to collect, prepare, read and quantify malaria smears by light microscopy.

\section{Setting}

Malaria transmission in Yaoundé is year round with four climatically distinct seasons composed of 2 short and two long dry and raining seasons respectively. Peak transmission occurs in the beginning of the rainy season. This assessment was conducted in April when transmission of malaria was expected to be at its peak.

\section{Specimen collection}

In each centre, $5 \mathrm{ml}$ of whole blood was collected from consenting febrile patients by venous puncture into prelabelled EDTA tubes. For each blood specimen, about $200 \mu \mathrm{l}$ was used to prepare a thick film and a thin blood smear for malaria parasite density determination and speciation. Microscopy was used as the reference test. This test was chosen because it is considered as the reference method for malaria diagnosis by the WHO. Blood smears were stained with freshly prepared $3 \%$ Giemsa solution according to standard procedures. To confirm microscopy diagnosis at the health centre, a new diagnosis was performed by two experienced and certified microscopists from the Biotechnology Centre and the Yaoundé University Teaching Hospital. They were 
blinded to the health centre diagnosis. The thick smears were used to detect the presence of Plasmodium infection. The thin smears were considered negative if no parasite was seen in 100 oil-immersion fields from two independent readings. A third reading was performed in case of positive/negative discordance for asexual stages.

\section{Rapid diagnostic tests}

The RDTs in the study were obtained from major local distributors of clinical rapid diagnostic tests and other clinical laboratory suppliers in Cameroon. In total, twenty-two different RDTs used for malaria diagnosis and present in the market in 2010 were obtained. Selection of the RDTs was based on their presence in the Cameroon market and distributor's willingness to participate in the assessments. The test cassettes or strips were of traceable quality (standard supplier, used within the shelf life of the product). History of proper storage and transport conditions from manufacturers could not be guaranteed.

The technical specifications of the various RDTs are presented as supplemental material (Additional file 1). Nine of the RDT kits detect only Plasmodium falciparum, while the rest had detection analytes for $P$. falciparum and PAN representing other Plasmodium species. The test devices were mostly plastic lateral flow cassettes (18) and dipsticks (4). Most of the RDTs kits contained specimen transfer/reagent transfer devices except stated in the instructions to use a specific transfer device like a micropipette. Test antigens used in the kits were $P$. falciparum specific histidine rich protein II (HRP2), lactate dehydrogenase (LDH) and aldolase in order of frequency of occurrence in RDTs.

\section{RDT test procedure and evaluating test results}

Evaluation of the 22 RDTs was performed and interpreted according to the manufacturers' instructions presented in the inserted inserts. Three technicians performed the tests. These technicians were not trained, but could interpret the procedures stated in the insert. In the case where the procedure required prior preparation of reagents or the use of multiple devices, each procedure technician consistently performed the same task each time to ensure uniformity in test performance. They were blinded to each other's results and to the results of microscopy. For each RDT the result was classified as negative, positive or invalid (Fig. 1). All tests without a control line were considered invalid by the test reader and the test repeated with a new RDT of the same code.

\section{Assessment of ease of use and interpretation of RDTs}

The study laboratory technicians who performed the RDTs ranked the 22 tests independently in order of preference where 2 corresponded to their most preferred

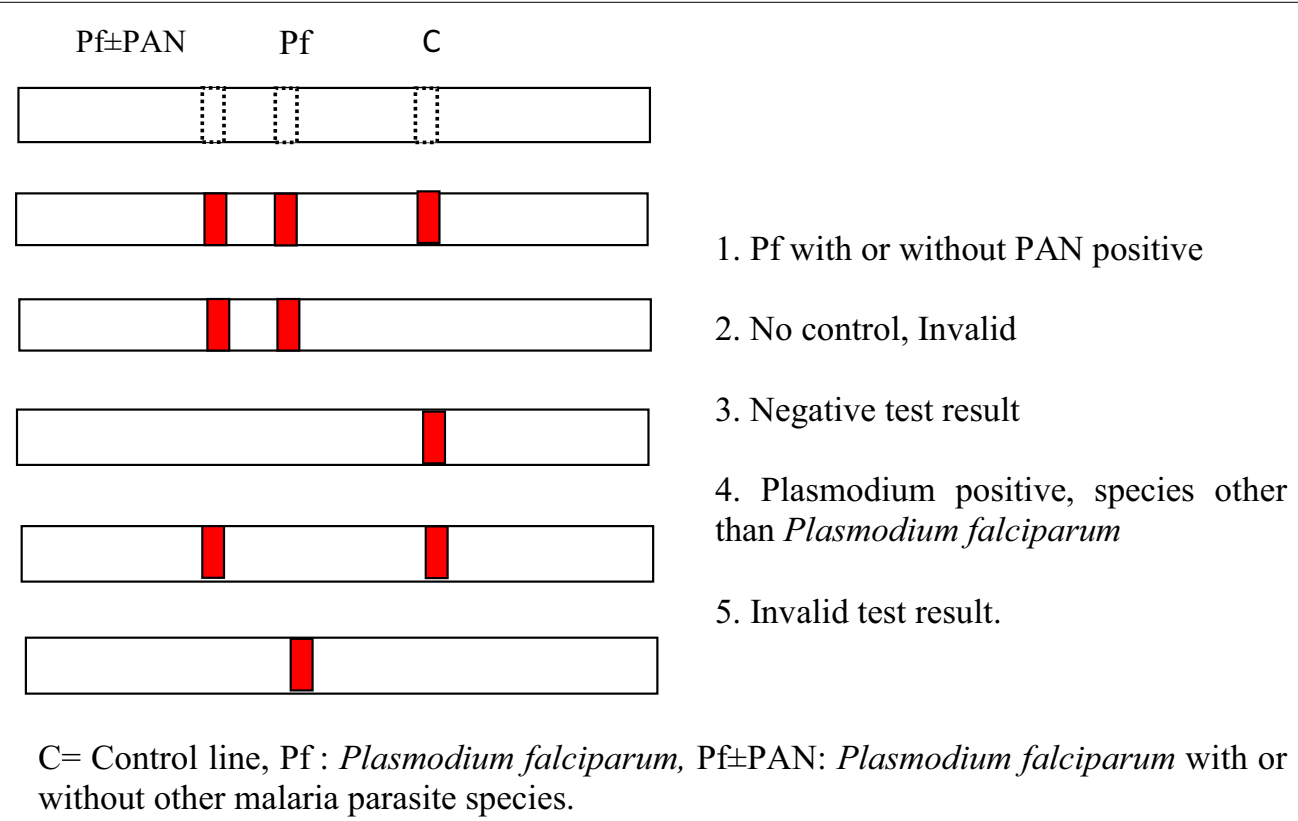

Fig. 1 Test line configuration for HRPII based RDTs used in our assessments and their interpretation. Line configurations differed slightly depending on target antigen. In this study target antigens for RDTs included Plasmodium LDH, HRP2 and aldolase. Invalid test cassettes were noted and replaced during the assessments. C: Control line, Pf: Plasmodium falciparum, Pf + PAN: Plasmodium falciparum with or without other malaria parasite species 
test and "0" to their least preferred based on a questionnaire response on a Likert scale from 0 (poor) to 3 (good) to 5(best): Ease and safety of taking blood; ease of adding reagents including the complexity of material/handling/ transfer technique and device required and ease of interpretation of insert instructions. In addition, assessors of RDT results were asked to rank the tests independently based ease of interpretation defined by the position of bands, labeling, band intensity, background and time to interpretation of results.

\section{Sample size, data management and analysis}

This assessment was a passive case detection survey. For malaria RDT under the study setting to perform well, the sensitivity should be at least $90 \%$ and the specificity $80 \%$. expert opinion was sought that indicated a likelihood ratio of the positive test of at least 2 was clinically useful in ruling in malaria diagnosis with a good RDT. The likelihood ratio was used as our as our diagnostic test index because it does not change with pre-test probability. Therefore the $95 \%$ confidence interval to exclude the likelihood ratio estimate using the values of the parameters provided above (Sensitivity/1-specificity) was calculated. This is given by the expression

$$
\mathrm{LR}_{x}=\exp \left(\operatorname{In} \frac{p_{1}}{p_{2}} \pm 1.96 \sqrt{\frac{1-p_{1}}{p_{1} n_{1}}+\frac{1-p_{2}}{p_{2} n_{2}}}\right)
$$

where $P_{1}$ and $P_{2}$ are sensitivity and (1-specificity) respectively and $\mathrm{n} 1=\mathrm{n} 2=$ sample size. In this way the sample size was estimated at 100 .

The data was checked for inconsistencies in data entry such as missing values and then used to calculate the sensitivity, specificity, the predictive values of the negative and positive RDT tests as well as the likelihood ratios and their $95 \%$ confidence intervals. Inter-observer variations in the RDT test assessments were estimated by calculating for each operational parameter the Cohen's kappa (к) statistic as follows:

$$
\text { Kappa }=\frac{A-B}{C-B}
$$

where $\mathrm{A}=$ agreement observed, $\mathrm{B}=$ agreement by chance $=50 \%$ of times, $\mathrm{C}=$ agreement possible $=100 \%$.

A $95 \%$ confidence interval ( $95 \%$ CI) was calculated for each $\kappa$ value and the overall agreement was evaluated as the weighted $\mathrm{k}$ value with its $95 \%$ confidence interval.

\section{Ethics statement}

This study was carried out under the auspices of the National Malaria Control Program with ethical clearance from the national ethics committee on health research in humans. Febrile patients whose left over blood specimen from routine medical exam were used provided verbal consent by phone. Information about the research was explained to each of one hundred participants/guardians by the consultant of the health centre either by phone or face to face for those who could come to the clinics. Participation was entirely voluntary.

\section{Results and discussion}

Overall, each of 22 different RDTs were tested on a total of ninety-seven venous blood samples. Three of the RDT test types were dipsticks while the rest were strips in rectangular cassettes.

\section{Sensitivity, specificity and predictive values of the rapid tests}

Results of the RDT evaluation are presented in Additional file 2. From this table, of all RDTs tested, (21/22) $95.4 \%$ had sensitivity values greater than $90 \%$ consistent with the WHO recommendations [7]. Among these RDTs with greater than $90 \%$ sensitivity, 7/9 $(77.8 \%)$ were "Pf Only" RDTs while the rest (14/22; $63.6 \%)$ were "Pf + PAN" RDTs. with sensitivity values greater than $90 \%$ indicating that there seems to be more of highly performing "Pf Only" RDTs than "Pf + PAN" RDTs Furthermore, only 6/13 (46.1\%) of "Pf + PAN" RDTs had sensitivity values less than $80 \%$. Two "Pf + PAN" RDTs had sensitivity less than $55 \%$. Considering specificity, only one "Pf Only" RDT had specificity greater than $90 \%$. The "Pf Only" RDTs had poor specificities with values around $60 \%$. On the other hand, only about 8 (36.3\%) "Pf + PAN" RDTs had sensitivity values greater than $90 \%$. Generally, RDTs with high sensitivity had low specificities and vice versa. A few RDTs (6/23 or $23 \%$ ) had sensitivities and specificity values of at least $80 \%$ and these were all "Pf + PAN" RDTs indicating that these RDT types seems to be better in diagnosing malaria when it is important to know if other species are contributing to the infection. Among all RDT analytes, the pLDH consistently showed higher sensitivity and specificity compared to the pHRP2. The predictive values of the positive test were generally below $80 \%$ for 18 RDTs $(78.2 \%)$ among which only 2 were "Pf Only" RDTs. Of all RDTs tested, 11(47.8 \%) had predictive values of the negative test greater than $80 \%$. Of these 11 RDTs, only one was a "Pf Only" RDT while among the "Pf + PAN" RDTs with greater than $80 \%$ predictive value of the negative test, only $1(7.6 \%)$ was represented by the pHRP2 analyte, comparing pHRP2 and pLDH/pAldolase on the "Pf + PAN" RDTs. This indicates that on the "Pf + PAN" RDTs, the PAN analyte on "Pf + PAN" RDTs seems to be better in excluding infections with $P$. falciparum compared to pfHRP2 on the same RDTs.

The majority of RDTs with good sensitivity had specificity less than $75 \%$. Only $6 / 14$ or $42 \%$ had sensitivity 
and specificity values greater than $80 \%$. Even though the sensitivity of an RDT is most often considered as the key parameter in clinical case management in malaria, it is desirable that the test analyte detects the antigens for which it is intended. One of the challenges associated with the use of RDTs is cross reactivity with antigens of a similar nature but from a different source [18]. Arguably, that the use of RDTs in case management provides added benefit of identifying patients who would otherwise have been missed if diagnosis relied on microscopy alone [19] eliminating the problem of overdiagnosis. Indeed, a recent exit poll involving malaria treated patients in Cameroon observed that over $50 \%$ of patients were overtreated for malaria [5]. It is important that the RDTs used for widespread identification of malaria patients within integrated programmes have good specificity values to avoid a negative impact of the implemented RDT for facility and community management of fevers.

\section{Performance of analytes in RDTs}

Tests that target HRP of $P$. falciparum demonstrated higher parasite detection rates compared to other analytes among all RDTs in this evaluation. However, tests that target LDH performed better when comparing both sensitivity and specificity for "Pf + PAN" RDTs. These findings are in agreement with the WHO based evaluation of RDTs for informed recommendations [7]. Therefore, tests that target HRP2 present in "Pf Only" RDTs appear to be better suited for the purpose of detecting the majority of malaria infections in the community. Furthermore, tests that were PAN specific had better predictive values of the positive and negative tests as well as the likelihood ratios of positive and negative tests compared to tests that detected HRP2 only. In the case where the additional objective would be to identify the presence of other species of parasites, test systems that include $\mathrm{pLDH}$ in association with HRP2 in this evaluation will be preferable. Plasmodium falciparum is the dominant species in our geo-ecological settings, but mixed parasite populations have been observed previously by our group to be circulating in some regions. In Bangolan, NW Cameroon where this observation was made, infections either consisted of $P$. falciparum coexisting with Plasmodium malariae (in $70 \%$ of cases) or non-P. falciparum (P. malariae in $30 \%$ of cases) [23]. Therefore, using a "Pf Only" RDT based on detection of HRP2 will miss $30 \%$ of single infections with non-falciparum parasites. In addition, there is the possibility of gene deletion isolates that do not express HRP-2 [20], although it has not been examined among parasite isolates from Cameroon and the same evidence for $\mathrm{pLDH}$ has not yet been found.

\section{Rapid diagnostic test agreement}

The weighted agreement between the two observers involved in reading the RDT results was quantified as the weighted Kappa value calculated for all test results irrespective of whether the test was a "Pf Only" or "Pf + PAN" RDT type. The weighted Kappa value obtained was $67.5 \%$ (95 \% CI 64.6-70.3 \%), corresponding to a moderately agreement between the assessors under our field condition. However, the agreements were better for some tests over others. For example, good agreement was obtained for more "Pf + PAN" RDTs than for "Pf Only" RDTs. SD Bioline Pf + PAN test cassette had the best agreement (72.6, 95 \% CI 58.9.6-86.4), based on the detection of both Pf and PAN while Clearview Malaria Combo was worst (54.9, 95 \% CI 38.8-71.2).

\section{Operational characteristics}

Based on the assessment of operational and technical characteristics of the 22 RDTs, differences in the test presentation, package contents, blood lancing and transfer devices, variations in insert instructions of use and safety disposal, procedures for test performance and results interpretation were observed. Additional file 3 provides a classification fo RDTs based on some operational characteristics. With the plethora of RDT tests commercially available today, even tests pre-qualified by the WHO and used in this assessment are very different in terms of their presentation, increasing the risk of error by the end user. Challenges ranging from the quality of information in inserts, configuration of test and control lines, missing information or components in the test kit, etc. all pose serious deficits that adversely influence the performance of RDTs at point of care and have also been reported recently [22]. Therefore, it is highly recommended that while efforts are being made to increase access of RDTs at the point of care, finding a way of providing a simple, informed and systematic presentation guideline and test to be followed by manufacturers of malaria RDTs destined for endemic countries is important.

Because of the free distribution of long-lasting insecticide-treated bed nets since 2011 to almost every household in Cameroon, a rapid decrease in the prevalence of malaria in many areas in Cameroon is expected. Low intensity infections may pose a diagnostic challenge for malaria surveillance purposes in the future due to shifting disease control priority, necessitating nucleic acid based tests.

\section{Study limitations}

This study has several potential limitations. Firstly, the results of rapid diagnostic tests vary as a function of parasite density [18]. It would have been valuable to classify 
RDTs based on parasite density to better compare results with the WHO standards. In addition, the results also assumed manufacturer reported stability. The present assessments were performed during a season when the minimal temperature in Yaoundé varied between 25 and $29{ }^{\circ} \mathrm{C}$. Further studies in which difference RDT performances will be compared in different real life environmental conditions will be very helpful to evaluate other stability parameters affecting the performance and decay of RDTs.

\section{Conclusion}

Of all RDTs tested, $94.2 \%(21 / 22)$ had sensitivity values greater than $90 \%$ among which 14 (63.6\%) were 'Pf + PAN' RDTs. The specificity was generally lower than the sensitivity for all RDTs, and poorer for "Pf Only" RDTs. The predictive values and likelihood ratios were better for non-HRP2 analytes for "Pf + PAN" RDTs. Rapid diagnostic tests which incorporate histidine rich protein II and lactate dehydrogenase are appropriate for settings. There was moderate agreement that SD Bioline Malaria Antigen P.f/Pan, ACON Malaria P.f/pan and Parascreen pf + PAN and CareStart ${ }^{\mathrm{TM}}$ Malaria HRP2/ pLDH Combo Test were most preferred in order of merit among "Pf + PAN" RDTs while SD Bioline Malaria Antigen P.f and ParaHIT ${ }^{\circledR} p f$ HRPII the most preferred among "Pf Only" RDTs. Among the dipsticks, "Pf + PAN" ParaHIT Total Dipstick was preferred. Harmonizing RDT test presentation and procedures would prevent mistakes of test performance and interpretation.

\section{Additional files}

Additional file 1: This Table represents the different RDTs present in the Cameroonian Market and used to evaluate accuracy under field conditions. Nineteen of the RDTs were of the lateral flow format while two were dipsticks. The assignment of codes for each RDT was purely random and this was done by an independent team member not involved with either performing the test or the reading of RDT test results.

Additional file 2: This table presents the aggregate results of the accuracy of rapid tests evaluated in the study.

Additional file 3: This table shows a classification of RDTs on the basis of some operational characteristics. Assessment of the suitability for field use included the simplicity of performing the test based on the insert instructions and illustrations on the one hand and the ease of interpretation of results within the time frame stated in the insert. The provision or requirement of additional materials like testubes, pipettes etc. were also taken into consideration in the final assessments. The scale used for classifying RDTs based on operational characteristics is alsho shown.

\section{Abbreviations}

RDT: rapid diagnostic test; pLDH: plasmodium lactate dehydrogenase; HRP2: histidine rich protein II; WHO: World Health Organization; PPV: positive predic tive value; NPV: negative predictive value.

\section{Authors' contributions}

RL, WM and DA conceived and designed the assessments and scored the final RDTs. IMA alongside JB, DA, FC, VT, NB, NP, JF, VA, NT performed the assessments. IMA, DA, and FCN analyzed the data. IMA drafted the manuscript and together with JB and DA. FCN and RL critically reviewed manuscript, alongside JM, ASE and EF. All authors reviewed and approved the final version of the manuscript before submission. All authors read and approved the final manuscript.

\section{Author details}

${ }^{1}$ The Biotechnology Centre, University of Yaoundé 1, BP 8094, Yaoundé, Cameroon. ${ }^{2}$ Department of Biochemistry, University of Dschang, Dschang, Cameroon. ${ }^{3}$ National Malaria Control Program, Ministry of Public Health, Yaoundé, Cameroon. ${ }^{4}$ Department of Biochemistry and Molecular Biology, University of Buea, Buea, Cameroon. ${ }^{5}$ Medical Research Centre, Institute of Medical Research and Medicinal Plant Study, Yaoundé, Cameroon. ${ }^{6}$ Faculty of Medicine and Biomedical Sciences, University of Yaoundé 1, Yaoundé, Cameroon. ${ }^{7}$ Ministry of Public Health, Yaoundé, Cameroon. ${ }^{8}$ Faculty of Health Sciences, Université des Montagnes, Bangante, Cameroon. ${ }^{9}$ Paediatrics Unit, University Teaching Hospital, Faculty of Medicine and Biomedical Sciences, University of Yaoundé 1, Yaoundé, Cameroon. ${ }^{10}$ Laboratory of Immunology and Parasitology, The Biotechnology Centre, University of Yaoundé 1, BP 8094, Yaoundé, Cameroon.

\section{Acknowledgements}

We wish to acknowledge all volunteers that participated by providing samples used for the evaluation. We equally acknowledge the District officer and chief medical officer as well as the staff of the Mfou District Hospital where the RDT assessments were carried out. Funds for the evaluation of the present RDTs came partly from contributions of the Global Fund Round 5 malaria funding to Cameroon.

\section{Competing interests}

The authors declare that they have no competing interests.

Received: 7 June 2015 Accepted: 8 January 2016

Published online: 20 January 2016

\section{References}

1. Gething PW, Patil AP, Smith DL, Guerra CA, Iqbal RFE, Johnston GL, et al. A new world malaria map: Plasmodium falciparum endemicity in 2010. Malar J. 2010;10:378.

2. WHO. World Malaria Report 2011. Geneva, World Health Organization, 2011. http://www.who.int/malaria/world_malaria_report_2011/worldmalariareport2011.pdf.

3. Kofoed PEL, Dias F, Lopes F, Rombo L. Diagnosis of fever in Africa. Lancet. 1998;351:373-4.

4. Reyburn H, Mbatia R, Drakeley C, Carneiro I, Mwakasungula E, et al. Overdiagnosis of malaria in patients with severe febrile illness in Tanzania: a prospective study. BMJ. 2004;329:1222.

5. Mangham LJ, Cundill B, Achonduh OA, Ambebila JN, Lele A, Metoh TN, et al. Malaria prevalence and treatment of febrile patients attending health facilities in Cameroon. Trop Med Int Health. 2011;17:330-42.

6. Thiam S, Thior M, Faye B, Ndiop MD, DioufML Diouf, et al. Major reduction in anti-malarial drug consumption in Senegal after nation-wide introduction of malaria rapid diagnostic tests. PLoS ONE. 2010;6:e18419.

7. Bell D, Wongsrichanalai C, Barnwell JW. Ensuring quality and access for malaria diagnosis: how can it be achieved? Nat Rev Microbiol. 2006:4:682-95.

8. WHO. The use of malaria rapid diagnostic tests. 2nd ed. Geneva: World Health Organization; 2006 (WHO-TDR/WHO-WPRO 2006).

9. Bjorkman A, Martensson A. Risks and benefits of targeted malaria treatment based on rapid diagnostic test results. Clin Infect Dis. 2010;51:512-4.

10. Greenwood BM, Armstrong JRM. Comparison of two simple methods for determining malaria parasite density. Trans R Soc Trop Med Hyg. 1991;85:186-8.

11. Plowe CV, Djimde A, Bouare M, Doumbo O, Wellems TE. Pyrimethamine and proguanil resistance-conferring mutations in Plasmodium falciparum dihydrofolate reductase: polymerase chain reaction methods for surveillance in Africa. Am J Trop Med Hyg. 1995;52:565-8. 
12. Mc Combie SC. Treatment seeking for malaria: a review of recent research. Soc Sci Med. 1996;43:933-45.

13. The malERA. Consultative Group. Diagnosis and diagnostics. A research agenda for malaria eradication: diagnoses and diagnostics. PLoS Med. 2011; 8:e1000396.

14. Yoel L, Hugh R, Hilda M, Rose M, Semkini C, Christopher JMW, et al. The impact of response to the results of diagnostic tests for malaria: costbenefit analysis. BMJ. 2008;336:202-5.

15. Lubell Y, Reyburn H, Mbakilwa H, Mwangi R, Chonya K, Whitty CJM, et al. The cost-effectiveness of parasitological diagnosis for malariasuspected patients in an era of combination therapy. Am J Trop Med Hyg. 2007;77:128-32.

16. Msellem MI, Martensson A, Rotllant G, Bhattarai A, Strberg J, Kahigwa E, et al. Influence of rapid malaria diagnostic tests on treatment and health outcome in fever patients, Zanzibar: a crossover validation study. PLoS Med. 2009;6:e1000070.

17. Zurovac D, Rowe AK, Ochola SA, Noor AM, Midia B, English M, et al. Predictors of the quality of health worker treatment practices for uncomplicated malaria at government health facilities in Kenya. Int J Epidemiol. 2004;33:1080-91.
18. Wongsrichanalai C, Barcus MJ, Muth S, Sutamihardja A, Wernsdorfer WH. A review of malaria diagnostic tools: microscopy and rapid diagnostic test (RDT). Am J Trop Med Hyg. 2007;77:119-27.

19. Baker J, McCarthy J, Gatton M, Kyle DE, Belizario V, Luchavez J, et al. Genetic diversity of Plasmodium falciparum histidine-rich protein 2 (PfHRP2) and its effect on the performance of PfHRP2-based rapid diagnostic tests. J Infect Dis. 2005;192:870-7.

20. Hopkins H, Bebell L, Kambale W, Dokomajilar C, Rosenthal PJ, Dorsey G. Rapid diagnostic tests for malaria at sites of varying transmission intensity in Uganda. J Infect Dis. 2008;197:510-8.

21. Graz B, Willcox M, Szeless T, Rougemont A. "Test and treat" or presumptive treatment for malaria in high transmission situations? A reflection on the latest WHO treatment guidelines. Malar J. 2010;10:136.

22. Jacobs J, Barbé B, Gillet P, Aidoo M, Serra-Casas E, Van Erps J, et al. Harmonization of malaria rapid diagnostic tests: best practices in labelling including instructions for use. Malar J. 2014;13:505.

23. Achonduh OA, Ekollo Mbange AH, Atogho-Tiedeu B, Ali IM, Achinko D, et al. Predominance of Plasmodium malariae-falciparum co-infection by molecular speciation in Bangolan, North West Region of Cameroon. J Life Sci. 2013;7:599-606.

\section{Submit your next manuscript to BioMed Central and we will help you at every step:}

- We accept pre-submission inquiries

- Our selector tool helps you to find the most relevant journal

- We provide round the clock customer support

- Convenient online submission

- Thorough peer review

- Inclusion in PubMed and all major indexing services

- Maximum visibility for your research

Submit your manuscript at www.biomedcentral.com/submit
() Biomed Central 